tention des amis de la Croix rouge. Nous espérons qu'il s'en trouvera parmi enx pour seconder les efforts de l'Institut de droit international, el pour user de leur influence en faveur de l'introduction dn Manuel dans les armées de leurs pays respectifs. Quand on s'est intéressé au sort des blessẻs, on ne peut rester indifférent à celui des autres victimes de la guerre.

\title{
ITALIE
}

\section{CIRCUlaire AUX COMITÉS ITALIENS}

Rome, 22 juillet 1880 .

\section{Monsieur le Président,}

La P'ésidence du Comité central est heureuse de pouvoir vous annoncer que la Croix rouge italienne aura celte année l'honneur de prendre part aux grandes manœuvres de l'armée.

L'importance de ce fait n'échappera certainement pas à Messieurs les membres des Comités de section et des Sous-Comités communaux du royaume.

Vous feriez chose utile, Monsieur le Président, en appelant sur ce point l'attention de Messieurs les sociétaires au moyen d'une réunion particulière, et celle du public par la voie de la presse.

Les progrès de notre grande association, pendant ces dernières années, pour son organisation intérieure autant que pour son développement économique et organique, lui ont mérité le témoignage de haute confiance que le ministre de la guerre a bien voulu lui accorder.

Le Comité local si méritoire des chevaliers italiens de l'Ordre de Malte, d'accord avec les prescriptions du ministre de la guerre, transportera à Gattinara (Haut-Novarais) sa baraque-hôpital, avec tout le matériel de secours et le personnel sanitaire dont il dispose.

Ce sera donc un premier essai, qui permettra de juger de l'utilité des hòpitaux ambulants et des moyens d'en diriger le service en temps de guerre. 
Le Comité des chevaliers italiens de l'Ordre de Malte représentera la Croix rouge italienne au champ des grandes manœuvres.

L'Association toute entière doil lui en être reconnaissante; nonseulement il s'astreint à une tâche difficile, mais il se charge d'une lourde responsabilité, puisqu'il ne s'agit de rien moins que de porter, sur le champ ou nos braves soldats accomplissent leurs importants exercices, les forces disciplinées de la charité sous le drapeau de la Croix rouge.

Le Comité central a pleine contiance dans les directeurs de l'ambulance des chevaliers, qui sentent, non moins que leurs collègues des autres nations, l'importance et l'élévation de la mission confiée à la Croix rouge.

Le sérienx et l'esprit d'examen qui ont présidé à l'organisation du matériel et au choix du personnel, sous la direction des chevaliers les plus distingués de l'Ordre et d'habiles médecins, garantissent un excellent résultat.

Nous croyons aussi que l'esprit d'abnégation et les sentiments généreux, qui animent la direction et le personnel sanilaire de la baraque-hôpital, gagneront la sympathie de notre glorieuse armée et la bienveillance de ses illustres chefs.

Une représentation de la Présidence se rendra au camp de Gattinara; ce sera là une bonne occasion pour étudier le fonctionnement de la Crojx rouge en campagne.

Le secrétaire général s'y rendra aussi, et c'esl à lui que les Comités italiens pourront adresser les lettres on les objets de secours et de soulagement dont on voudrait faire l'essai.

La baraque-hòpital sera en exercice près du camp de Gattinara du 28 aoùt au 11 septembre 1880.

Le rapport du directeur sanitaire de la baraque-hòpital, après les grandes manœuvres, sera inséré dans le Bulletin de l'Association. C'est pour cette raison que sa publication en sera retardée jusqu'au mois de septembre prochain.

Recevez, etc.

Pour le Comité central de la Croix rouge:

Le Président,

Commandeur Enrico Guicciardi,

Le Secrétaire général, Sénateur du royaume. Vincenzo Maggionani. 